\title{
Variabilidad hidroclimática histórica de la sierra de Zapalinamé y disponibilidad de recursos hídricos para Saltillo, Coahuila
}

\author{
Historical hydroclimatic variability of the Zapalinamé Sierra \\ and water resources for Saltillo, Coahuila
}

\author{
José Villanueva Díaz¹, Julián Cerano Paredes¹, \\ Vicenta Constante García1, Peter Z. Fulé2 \\ y Eladio Cornejo Oviedo3
}

\begin{abstract}
RESUMEN
La Sierra de Zapalinamé (SZA) es la fuente de agua más importante para Saltillo, Coahuila y poblaciones aledañas. La presión sobre los recursos hídricos debido al incremento poblacional, demanda un conocimiento detallado de su variabilidad hidroclimática histórica con fines de planeación. En este trabajo, se integraron tres series dendrocronológicas regionales a lo largo del gradiente altitudinal de SZA, con una extensión de 412 años (1595-2006), 306 años (1700-2005) y 603 años (1400-2002), para la parte baja, media y alta, respectivamente. La cronología de la parte baja, desarrollada con Pinus cembroides fue más sensible a periodos secos. Sequías de gran magnitud e intensidad se presentaron en todo el gradiente altitudinal en las décadas de 1440 y 1450; 1530, 1590, $1640,1660,1690,1710,1740,1750,1760,1790,1860,1890,1920,1950$ y 2000 , con impacto en la disponibilidad de alimentos y presencia de epidemias y hambrunas generalizadas a nivel local y regional. Eventos secos de alta intensidad se presentaron en periodos aproximados de 100 años, con sequías intermedias cada 50 años. El impacto histórico de patrones atmosféricos circulatorios como ENSO, no indicó una influencia significativa en la variabilidad hidroclimática de SZA y el efecto de otros fenómenos atmosféricos (frentes fríos, huracanes, tormentas tropicales), pudieran explicar con mayor detalle el comportamiento climático de esta montaña.
\end{abstract}

PALABRAS CLAVE:

Dendrocronología, hidroclima, Pinus cembroides, sequía, Sierra de Zapalinamé.

\begin{abstract}
The Sierra Zapaliname (SZA) is the most important source of water for Saltillo and neighboring settlements. Population increase is putting pressure on limited water sources, therefore it is necessary to have a better understanding of historical hydroclimate variability for planning the proper use of limited water resources. Tree-ring series were developed for the lower, mid, and upper sections along an altitudinal gradient in SZA. The chronology length was 412 years (1595-2006), 306 years (17002005), and 603 years (1400-2002), for the lower, mid, and higher elevation, respectively. The lower elevation tree-ring chronology had higher climatic sensitivity to dry periods as compared to those of the

Instituto Nacional de Investigaciones Forestales, Agrícolas y Pecuarias. Centro Nacional de Investigación Disciplinaria Relación Agua-Suelo-Planta-Atmósfera. Km 6.5 margen derecha Canal Sacramento Gómez Palacio, Dgo. Apdo. Postal 41, Código Postal 35150. Lerdo, Dgo. villanueva.jose@inifap.gob.mx, cerano.julian@inifap.gob.mx, constante.garcia@inifap.gob.mx Northern Arizona University; School of Forestry and Ecological Restoration Institute. P. O. Box 1508. Flagstaff, AZ 86011, USA. pete.fule@nau.edu Universidad Autónoma Agraria “Antonio Narro”, Buenavista, Saltillo, Coahuila. cor61@prodigy.net.mx.
\end{abstract}


mid and upper elevation sections. Intense and extended droughts were detected along the altitudinal gradient in the $1440 \mathrm{~s}$ to $1450 \mathrm{~s}, 1530 \mathrm{~s}, 1590 \mathrm{~s}$, $1640 s, 1660 s, 1690 s, 1710 s, 1740 s$, $1750 \mathrm{~s}, 1760 \mathrm{~s}, 1790 \mathrm{~s}, 1860 \mathrm{~s}, 1890 \mathrm{~s}$, 1920s, 1950s, and 2000s, and produced local and regional food shortage and epidemic outbreaks. Severe droughts were detected at frequencies of 100 years followed by mid intensive droughts at 50-year periods. The climatic influence of ENSO in determining hydroclimatic behavior of SZA has not been significant and other circulatory patterns (cold fronts, hurricanes, tropical storms) could better explain the historical climate variability that has characterized this mountain range.

KEY WORDS:

Dendrochronology, hydroclimate, drought, Pinus cembroides, Sierra de Zapaliname

\section{INTRODUCCIÓN}

La conservación de ecosistemas de áreas con alta biodiversidad, que a la vez constituyen fuente importante de servicios hidrológicos, captura de carbono, ecoturismo, entre otros servicios ambientales que proporcionan a una región determinada, requiere de un conocimiento más detallado de la variabilidad hidroclimática en el tiempo y de su impacto en el ciclo hidrológico, tasas de crecimiento de las especies dominantes, así como de sus interrelaciones ecológicas con el resto de sus componentes.

La Sierra de Zapalinamé en el estado de Coahuila (SZA) es un área de conservación prioritaria, que independientemente de su riqueza biológica constituye la fuente más importante de recursos hídricos para los municipios de Saltillo, Arteaga y Ramos Arizpe, en el estado de Coahuila, a los cuales suministra un volumen de agua de $30 \times 10^{6} \mathrm{~m}^{3}$ año-1, que representa más del $50 \%$ del requerimiento total de agua potable que demanda esta urbe; requerimiento que actualmente supera los $70 \times 10^{6} \mathrm{~m}^{3}$ año-1
(Navarro, 2008). En la última década, el crecimiento poblacional de esta región ha originado la expansión del núcleo urbano, establecimiento de nuevas industrias $y$ demanda de más servicios urbanos; situación que ha incrementado la demanda de agua para consumo humano, uso industrial y agropecuario; volumen que es subsanado en parte con un incremento en el volumen de extracción de agua del acuífero, lo que acarrea problemas subsecuentes de abatimiento y de calidad de agua (Comisión Nacional del Agua, 2006).

El cambio de uso del suelo, efecto de incendios forestales de origen antropogénico, explotación de material pétreo, ampliación de la mancha urbana, recreación desordenada, desarrollos campestres; entre otras acciones derivadas de actividades humanas como son pastoreo, extracción de leña, madera y productos no maderables, constituyen acciones de disturbio que ejercen una fuerte presión en la estabilidad de los recursos bióticos y abióticos de la Sierra de Zapalinamé; acción que sobrepasa los esfuerzos de conservación y restauración de ecosistemas en los que están involucrados organismos de investigación, educación e instituciones gubernamentales y no gubernamentales (UAAAN, 1998).

No obstante lo anterior, la conservación de esta zona se considera de alta prioridad para el municipio de Saltillo, y estudios técnicos que contribuyan a un entendimiento del funcionamiento de sus ecosistemas resultan de alto valor científico, particularmente para el desarrollo de planes de manejo sustentable y de conservación de sus recursos naturales.

La Sierra de Zapalinamé conforma un gradiente altitudinal con la presencia de ecosistemas áridos en la parte baja, hasta bosque templado mixto en elevaciones medias y superiores. Las comuni- 
dades vegetales presentes en este gradiente poseen especies que presentan anillos de crecimiento anual bien definidos, situación que puede ser aprovechada con fines de reconstrucción dendroclimática, ya que algunos de los especímenes presentes en estas comunidades alcanzan edades que superan los 400 años de edad (Villanueva et al., 2006; Constante, 2007).

De esta manera, el objetivo del presente trabajo es utilizar los crecimientos anuales de algunas especies arbóreas como "proxy" de la variabilidad hidroclimática, que ha caracterizado a la SZA en todo su gradiente altitudinal para los últimos cuatro siglos, y analizar el efecto que esta variabilidad climática ha generado en aspectos económicos y sociales del núcleo poblacional de Saltillo, Coahuila.

\section{METODOLOGÍA}

El estudio se realizó en la SZA, que forma parte de la provincia fisiográfica de la Sierra Madre Oriental, subprovincia Gran Sierra Plegada. Posee un clima semiárido en su parte media y baja hasta templado en su parte alta y una precipitación que varía de $257 \mathrm{~mm}$ a más de $400 \mathrm{~mm}$ a lo largo de su gradiente altitudinal.

La vegetación dominante es del tipo matorral desértico micrófilo y rosetófilo en la parte seca, con bosques de pino piñonero en su parte media y de otras coníferas en su parte más alta (Marroquín y Arce, 1985). Hidrológicamente se ubica en las vertientes del Golfo de México y cuencas cerradas del norte y región hidrológica 24 (Bravo-Conchos) (Comisión Nacional del Agua, 2002).

En esta área, núcleos de crecimiento, virutas o gusanos y secciones transversales se obtuvieron con un taladro de Pressler y motosierra, de especímenes seleccionados de pino piñonero (Pinus cembroides) y de ayarín, cahuite o Douglas-fir (Pseudotsuga menziesii) ubicados en bosque de piñonero y bosque mixto de coníferas en la parte baja, media y alta de la SZA. En total se realizaron tres colectas de pino piñonero distribuidas en las localidades de Cuauhtémoc, Huachichil y El Diamante y una colecta de ayarín en el paraje "El Penitente".

Los núcleos de crecimiento, se identificaron, montaron y pegaron en secciones acanaladas de madera para facilitar su manejo; posteriormente éstas se pulieron intensamente con diversos grados de lija (grano grueso a fino); el mismo procedimiento fue utilizado con las secciones trasversales para resaltar sus estructuras de crecimiento. En el laboratorio de Dendrocronología del INIFAP CENID-RASPA, y con el apoyo de un estereomicroscopio trilocular de alta resolución, los anillos se contaron y fecharon al año exacto de su formación, mediante el uso de técnicas dendrocronológicas estándar (Stokes y Smiley, 1968). Una vez fechadas las muestras, cada crecimiento individual (anillo anual) de $P$. menziesii se seccionó en bandas de madera temprana ( $E W$, siglas en inglés), tardía (LW, siglas en inglés) y anillo total (RW, siglas en inglés) y posteriormente estas bandas se midieron de forma separada con un sistema de medición VELMEX (Robinson y Evans, 1980). Para el caso de $P$. cembroides sólo se midió anillo total (RW), debido a la escasa formación y dificultad para diferenciar y medir la madera tardía.

El fechado, calidad de la respuesta climática y exactitud de la medición de cada anillo, se verificó con el programa COFECHA (Holmes, 1983; GrissinoMayer, 2001). Las tendencias biológicas y geométricas no relacionadas con clima, 
se removieron con el programa ARSTAN, al insertar una curva exponencial negativa o línea recta a la serie de medición y luego al dividir cada valor anual de medición entre el valor obtenido de la curva (Cook, 1987).

Debido a la cercanía geográfica de las cronologías de SZA con otras previamente desarrolladas en Sierra de Arteaga (La Viga, Morro, Coahuilón y Los Pilares) en Coahuila y Peña Nevada, en Nuevo León, éstas se incluyeron para análisis comparativos con el fin de tener un conocimiento más detallado de la variabilidad climática histórica de la región (Figura 1). Para definir la respuesta climática entre cronologías, se desarrollaron correlaciones simples entre las cronologías; asimismo, se utilizó la subrutina Análisis de Componentes Principales (PCA por sus siglas en inglés) del programa STATISTICA Kernel release 5.5 (Stat Soft Inc., 2000), para definir aquellas series dendrocronológicas con respuesta común y de esta forma integrarlas en cronologías regionales más representativas de la variabilidad hidroclimática que caracteriza a la SZA en su parte baja y media y alta, respectivamente.

Los índices dendrocronológicos de las cronologías regionales se compararon entre sí y también con información documentada histórica relacionada con impacto de eventos climáticos extremos, en la producción de alimentos, índices de ENSO e influencia de otros fenómenos circulatorios como tormentas tropicales, nortes y lluvias convectivas. Para deter-

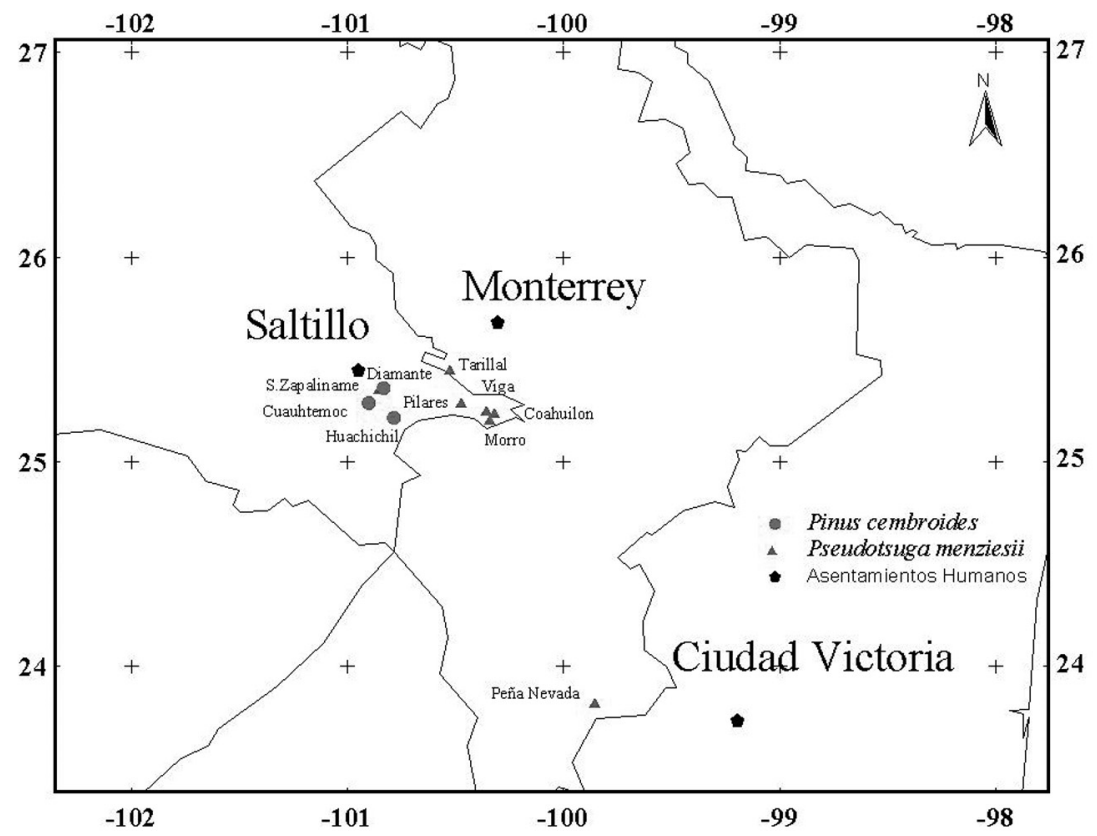

Figura 1. Distribución geográfica de cronologías de Pseudotsuga menziesii y Pinus cembroides desarrolladas para Sierra de Zapalinamé y sitios aledaños a esta montaña. 
minar la influencia del clima en el crecimiento estacional de $P$. cembroides, se recopilaron del extractor rápido de información climática (ER/C II, 2000) aquellas estaciones más cercanas a los sitios de colecta y se generó una serie de datos regionales (Tabla 1). La respuesta climática entre precipitación y crecimiento anual se investigó con el programa PRECON (Fritts, 1999).

Con el total de datos climáticos obtenidos se realizó una calibración entre la cronología y los registros estacionales de precipitación (Fritts, 1991). Finalmente, se obtuvo una ecuación de transferencia para el periodo total de datos de precipitación disponibles, la cual fue utilizada para desarrollar la reconstrucción de precipitación en la longitud total de la cronología. A la serie de alta frecuencia (resolución anual), se le ajustó una curva decenal flexible (baja frecuencia) para resaltar eventos secos o húmedos (Cook y Peters, 1981). Los periodos de sequía presentes en la reconstrucción se compararon con periodos similares detectados en otras reconstrucciones de precipitación realizadas para sitios ubicados en la Sierra Madre Oriental y Occidental, así como con la ayuda de archivos históricos, información de importancia para analizar la extensión de las sequías y los posibles mecanismos climatológicos comunes involucrados en determinar el comportamiento hidroclimático de estas regiones.

El análisis del impacto del fenómeno ENSO se analizó a través del Índice de Lluvia Tropical (TRI, por sus siglas en inglés), índice que constituye un estimativo de variación de ENSO, al constituir anomalías de precipitación en el Pacífico Central y que parece ser más estable que el Índice Tahiti-Darwin (Wright, 1979; Cleaveland et al., 2003).

\section{RESULTADOS}

\section{Ubicación y extensión de las cronologías}

Se generó un total de cuatro cronologías para la SZA, tres de ellas de Pinus cembroides ubicadas en elevaciones bajas y una de Pseudotsuga menziesii para la parte alta de la montaña. La ubicación y extensión de las cronologías previamente desarrolladas para Sierra de Arteaga y Peña Nevada se describen en la tabla 2.

Tabla 1. Estaciones meteorológicas consideradas para la realización del promedio de precipitación mensual regional.

\begin{tabular}{lcc}
\hline $\begin{array}{l}\text { Estación } \\
\text { Meteorológica }\end{array}$ & $\begin{array}{c}\text { Latitud } \\
\text { Norte } \\
\text { (grados) }\end{array}$ & $\begin{array}{c}\text { Longitud } \\
\text { Oeste } \\
\text { (grados) }\end{array}$ \\
\hline Arteaga, Arteaga & 25,43 & 100,85 \\
General Cepeda & 25,37 & 101,47 \\
Saltillo & 25,42 & 101,00 \\
San Antonio de las Alazanas (smn) & 25,28 & 100,62 \\
Gómez Farías & 24,97 & 101,05 \\
San Juan de la Vaquería & 25,25 & 101,22 \\
Huachichil, Arteaga & 25,23 & 100,83 \\
El Tunal, Arteaga & 25,42 & 100,63 \\
\end{tabular}


Tabla 2. Relación de cronologías de anillos de árboles desarrolladas para la Sierra de Zapalinamé y montañas aledañas.

\begin{tabular}{|c|c|c|c|c|c|c|}
\hline Estado & $\begin{array}{l}\text { Nombre del } \\
\text { Sitio }\end{array}$ & $\begin{array}{l}\text { Clave } \\
\text { del Sitio }\end{array}$ & $\begin{array}{l}\text { Coordenadas (grados) } \\
\text { y elevación }\end{array}$ & $\begin{array}{l}\text { Longitud } \\
\text { de Cronología } \\
\text { (años) }\end{array}$ & $\begin{array}{l}\text { Tipo de } \\
\text { Cronología } 1\end{array}$ & Especie $^{2}$ \\
\hline \multirow[t]{10}{*}{ Coahuila } & Viga $^{3}$ & VIG & $25,238,100,372 ; 3400 \mathrm{~m}$ & $1659-2001$ & RW, EW, LW & PM \\
\hline & Coahuilón ${ }^{3}$ & $\mathrm{COA}$ & $25,234,100,333 ; 3200 \mathrm{~m}$ & $1700-2001$ & RW, EW, LW & PM \\
\hline & Pilares 3 & PIL & $25,275,100,492 ; 3150 \mathrm{~m}$ & $1775-2000$ & RW, EW, LW & PM \\
\hline & Morro $^{3}$ & MOR & $25,201,100,355 ; 3500 \mathrm{~m}$ & $1855-2000$ & RW, EW, LW & $\mathrm{PM}$ \\
\hline & Tarillal3 & TAR & $25,440,100,551 ; 3200 \mathrm{~m}$ & $1872-2000$ & RW, EW, LW & PM \\
\hline & Zapalinamé4 & ZAP & $25,284,100,925 ; 2300 \mathrm{~m}$ & $1595-2004$ & RW & PC \\
\hline & Sierra de & & & & & \\
\hline & Zapalinamé4 & SZA & $25,35,100,90 ; 2,700 \mathrm{~m}$ & $1746-2005$ & RW, EW, LW & PM \\
\hline & Huachichil 4 & HUA & $25,208,100,825 ; 2087 \mathrm{~m}$ & $1869-2006$ & RW & PC \\
\hline & Diamante 4 & DIA & $25,352,100,876 ; 2280 \mathrm{~m}$ & $1845-2006$ & RW & $\mathrm{PC}$ \\
\hline Nuevo León & Peña Nevada5 & PNE & $23,817,99,841 ; 3200 \mathrm{~m}$ & $1400-2002$ & RW, EW, LW & PM \\
\hline
\end{tabular}

1RW: Ancho de anillo total; EW: Madera temprana; LW: Madera tardía; 2PM: Pseudotsuga menziesii; PC: Pinus cembroides; ${ }^{3}$ Cronologías desarrolladas en la Sierra de Arteaga, Coah.; ${ }^{4}$ Cronologías desarrolladas en Sierra de Zapalinamé, Coah.; 5 Cronología desarrollada en Peña Nevada, N.L.

\section{Asociación entre cronologías}

La asociación entre dos cronologías se definió estadísticamente mediante el coeficiente de correlación, estadístico que define la similitud o grado de correspondencia entre dos series de tiempo (Fritts, 1976). El análisis de correlación indicó una asociación altamente significativa $(p<0,0001)$ entre las series analizadas, lo cual es indicativo de que el crecimiento de los árboles en el gradiente altitudinal de SZA tiene una respuesta común a eventos climáticos que afectan esta montaña; lo cual es independiente de la cantidad de precipitación que se reciba en cada sitio, es decir, un evento de precipitación puede provocar más lluvia en la parte alta de la montaña, pero también producirá lluvia, aunque en menor proporción en su parte baja (Tabla 3).
A pesar de que las correlaciones entre cronologías fueron significativas, el Análisis de Componentes Principales definió dos factores (eigenvalues o valores característicos) dominantes con la mayor varianza posible, el primero de ellos, explica $62,5 \%$ de la varianza total e integra a las cronologías de Pseudotsuga menziesii localizadas a elevaciones superiores, entre las que se encuentra Peña Nevada (PNE), La Viga (VIG), El Coahuilón ( $\mathrm{COH})$, Los Pilares (PIL), El Morro (MOR), El Tarillal (TAR) y Sierra Zapalinamé (SZA). El segundo componente, que explica $11 \%$ de la varianza total incluye a cronologías de elevaciones bajas; es decir, las de Pinus cembroides, entre las que se encuentran las del ejido Cuauhtémoc (ZAP), Huachichil (HUA) y El Diamante (DIA) (Figura 2). 
Tabla 3. Correlaciones simples entre las cronologías de Sierra de Zapalinamé, Sierra de Arteaga y Peña Nevada, para un periodo común de 120 años (1872 - 2000).

1Coeficientes de correlación entre cronologías $(n=129 ; p<0.0001)$

\begin{tabular}{|c|c|c|c|c|c|c|c|c|c|c|}
\hline & PNE & ZAP & VIG & $\mathrm{COA}$ & PIL & MOR & TAR & HUA & DIA & SZA \\
\hline PNE & 1,00 & 0,43 & 0,60 & 0,56 & 0,51 & 0,43 & 0,52 & 0,25 & 0,35 & 0,55 \\
\hline ZAP & 0,43 & 1,00 & 0,61 & 0,67 & 0,52 & 0,44 & 0,48 & 0,65 & 0,82 & 0,65 \\
\hline VIG & 0,60 & 0,61 & 1,00 & 0,72 & 0,78 & 0,66 & 0,69 & 0,59 & 0,63 & 0,66 \\
\hline COA & 0,56 & 0,67 & 0,72 & 1,00 & 0,67 & 0,62 & 0,55 & 0,49 & 0,65 & 0,69 \\
\hline PIL & 0,51 & 0,52 & 0,78 & 0,67 & 1,00 & 0,56 & 0,73 & 0,51 & 0,48 & 0,63 \\
\hline MOR & 0,43 & 0,44 & 0,66 & 0,62 & 0,56 & 1,00 & 0,54 & 0,39 & 0,52 & 0,51 \\
\hline TAR & 0,52 & 0,48 & 0,69 & 0,55 & 0,73 & 0,54 & 1,00 & 0,41 & 0,49 & 0,72 \\
\hline HUA & 0,25 & 0,65 & 0,59 & 0,49 & 0,51 & 0,39 & 0,41 & 1,00 & 0,76 & 0,59 \\
\hline DIA & 0,35 & 0,82 & 0,63 & 0,65 & 0,58 & 0,42 & 0,49 & 0,76 & 1,00 & 0,71 \\
\hline SZA & 0,55 & 0,65 & 0,66 & 0,69 & 0,63 & 0,51 & 0,72 & 0,59 & 0,71 & 1,00 \\
\hline
\end{tabular}

1 La correlación entre cronologías fue altamente significativa $(p<0,0001)$ para todas las comparaciones realizadas; la correlación más alta detectada entre dos cronologías se indica en negritas.

\section{Factor Loadings Plot}

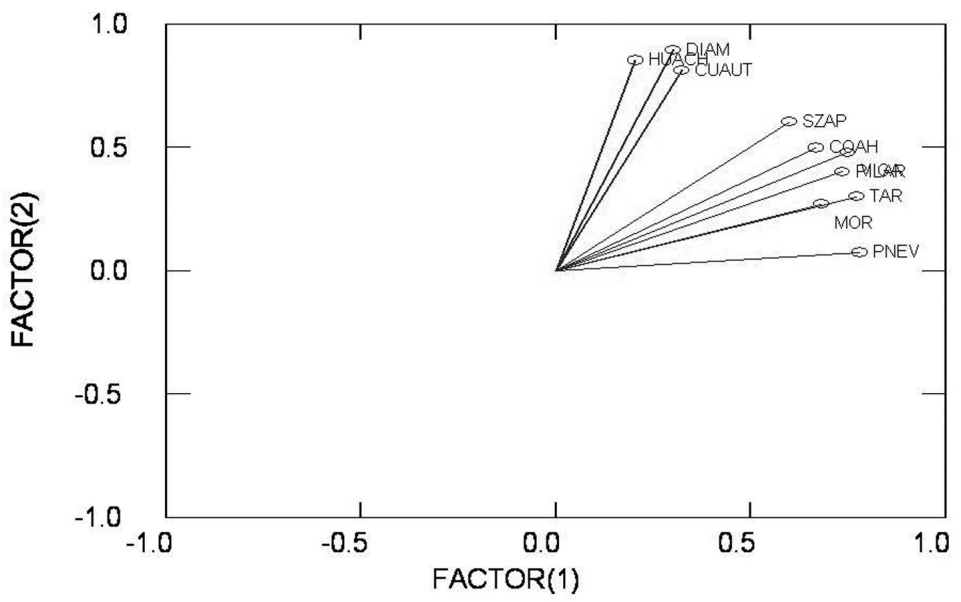

Figura 2. Agrupación de cronologías derivado del análisis de Componentes Principales. Se observan dos grupos definidos, uno para la parte baja integrado por las cronologías de Pinus cembroides (DIA, HAU, CUA) y otro más para la parte media y alta integrado por cronologías de Pseudotsuga menziesii (SZA, COAH, VIG, TAR, MOR, PNE). 
Con base en la presencia de grupos bien definidos y altamente correlacionados entre sí, se integraron tres cronologías climáticamente representativas de la parte baja, media y alta del gradiente altitudinal en SZA. Aunque el PCA determinó un solo grupo para la parte media y alta, se observó una alta correlación entre SZA y $\mathrm{COAH}$, que corresponden a la parte media, las cronologías restantes de la parte alta incrementaron su correlación al eliminar estas series, por esta razón se consideró generar tres cronologías. El primer grupo para la parte baja (>2 $300 \mathrm{~m}$ ) se integró con las cronologías ZAP, HUA y DIA; para la parte intermedia del gradiente (2 $700 \mathrm{~m}-3200 \mathrm{~m}$ ) con las cronologías COAH y SZA y para la parte elevada (3 200 m-3 600 m) VIG, PIL, TAR y PNE. La cronología MOR se eliminó de la parte alta por mostrar una correlación relativamente menor en comparación con el resto de cronologías y esta acción permitió generar una cronología de mayor calidad en términos de respuesta climática.
La asociación entre cronologías representativas de la parte alta, media y baja del gradiente altitudinal de la SZA fue significativa tanto para el periodo común de comparación (1700-2002) como para subperiodos de 50 años (Tabla 4). Las estadísticas descriptivas de las cronologías regionales muestran potencial adecuado para el desarrollo de reconstrucciones paleoclimáticas. Con base únicamente en la sensitividad media y desviación estándar, la cronología regional de la parte baja, derivada de Pinus cembroides indica mayor potencial para análisis de la variabilidad climática de la zona en comparación a las cronologías regionales de Pseudotsuga menziesii para las partes media y alta de la Sierra de Zapalinamé. El Pinus cembroides se desarrolla en sitios secos, sujetos a baja disponibilidad de agua; de esta manera la especie es más sensible a cambios en precipitación, que se reflejan en mayor variabilidad interanual en sus índices dendrocronológicos (Tabla 5).

Tabla 4. Correlaciones derivadas de comparaciones entre los índices de anillo total (IAA) de cronologías representativas de la parte alta, media y baja del gradiente altitudinal para el periodo común de comparación (1700 - 2002)

y para subperiodos de 50 años.

\begin{tabular}{lccc}
\hline Periodo & \multicolumn{3}{c}{ Cronologías regionales comparadas } \\
\cline { 2 - 4 } & Alta - Media & Media - Baja & Alta - Baja \\
\hline $1700-2002$ & 0,62 & $\mathbf{0 , 6 4 1}$ & 0,55 \\
$1700-1749$ & 0,29 & 0,57 & 0,37 \\
$1750-1799$ & 0,54 & 0,62 & $\mathbf{0 , 6 4 1}$ \\
$1800-1849$ & 0,62 & 0,49 & $\mathbf{0 , 6 4 1}$ \\
$1850-1899$ & $\mathbf{0 , 7 2 1}$ & $\mathbf{0 , 7 0 1}$ & 0,54 \\
$1900-1949$ & $\mathbf{0 , 7 6 1}$ & $\mathbf{0 , 6 9 1}$ & $\mathbf{0 , 6 4 1}$ \\
$1950-1999$ & $\mathbf{0 , 8 4 1}$ & $\mathbf{0 , 8 0 1}$ & 0,57 \\
\hline
\end{tabular}

1 Todos los valores de correlación fueron significativos $(p<0,005)$; los valores en negritas corresponden a las correlaciones más altas detectadas entre dos cronologías. 
Tabla 5. Estadísticas descriptivas de las cronologías regionales de anillos de árboles generadas por el programa ARSTAN para las series dendrocronológicas regionales de la parte baja, media y alta de Sierra de Zapalinamé, Coahuila.

\begin{tabular}{|c|c|c|c|c|c|c|}
\hline \multirow[t]{2}{*}{ Estadísticos } & \multicolumn{2}{|c|}{ Regional Baja } & \multicolumn{2}{|c|}{ Regional Media } & \multicolumn{2}{|c|}{ Regional Alta } \\
\hline & Estándar & Residual & Estándar & Residual & Estándar & Residua \\
\hline Media & 0,9841 & 0,9949 & 0,9775 & 0,9884 & 1,000 & 0,9952 \\
\hline Mediana & 0,9854 & 0,9868 & 0,9827 & 0,9970 & 0,9729 & 0,9927 \\
\hline Sensitividad Media & 0,3180 & 0,3475 & 0,2968 & 0,3335 & 0,2539 & 0,2561 \\
\hline Desviación Estándar & 0,3110 & 0,2924 & 0,3092 & 0,2902 & 0,3021 & 0,2248 \\
\hline 1Asimetría (Skewness) & 0,1209 & 0,1935 & 0,1069 & 0,0218 & 0,7072 & 0,0947 \\
\hline 2Kurtosis & $-0,1363$ & $-0,1638$ & $-0,2964$ & 0,2256 & 2,1209 & 0,1313 \\
\hline${ }^{3}$ Autocorrelación de $1 \mathrm{er}$ orden & 0,2871 & $-0,0496$ & 0,2625 & $-0,0685$ & 0,4309 & $-0,0013$ \\
\hline Autocorrelación de $2^{\circ}$. Orden & 0,1271 & 0,0295 & 0,1872 & $-0,0204$ & 0,1702 & 0,0000 \\
\hline Autocorrelación de 3er. orden & $-0,0494$ & $-0,0452$ & 0,0496 & $-0,0234$ & 0,0170 & $-0,0227$ \\
\hline
\end{tabular}

1Skewness es una medida de simetría en una distribución normal; 2Kurtosis se refiere a si los datos se concentran (hacen pico) o tienen un comportamiento más uniforme (aplanado) en una distribución normal; ${ }^{3}$ Autocorrelación es una medición de la tendencia de una especie arbórea a producir crecimientos similares de un año a otro, esta tendencia disminuye con el tiempo.

\section{Respuesta climática de las cronologías}

La cronología representativa de la parte baja se extiende para el periodo 1595-2006, e indica escaso crecimiento en ciertos periodos, relacionado con la presencia de eventos secos en las décadas de 1590, 1640, 1660, 1690, $1710,1740,1750,1760,1790,1860$, 1890, 1920, 1950 y 2000 (Constante, 2007; Santillán, 2008). Los índices más altos, relacionados con periodos húmedos fueron menos frecuentes y se presentaron en las décadas de 1630, 1720, 1770, 1840, 1930, 1960 y 1980 (Figura 3). La cronología estándar representativa de la parte media se extiende para el periodo 1700-2005 e indica sequías de gran magnitud que impactaron esta parte del gradiente, particularmente en las décadas de 1730, 1780, 1810, 1860, 1890, 1920, 1950 y 2000. Los periodos húmedos más relevantes se presentaron en las décadas de 1720 ,
1770, 1830, 1880, 1940 y 1980 (Figura 4). La cronología estándar representativa de la parte alta se extiende para el periodo 1400-2002 y muestra reducción en el índice normalizado de anillos de crecimiento, relacionado con periodos secos para las décadas de 1440 y 1450; 1530, $1580,1690,1720,1760,1810,1870$, 1890, 1920, 1950 y 1970. Periodos húmedos se detectaron en las décadas de 1460, 1540, 1650, 1740, 1830 y 1980 (Figura 5).

\section{Reconstrucción de precipitación}

Debido a la mayor sensibilidad climática de la cronología regional de la parte baja y a la similitud de respuesta climática entre las cronologías regionales (baja, media y alta de la SZA), se desarrolló una reconstrucción de precipitación estacional para definir en términos cuantitativos la variabilidad hidroclimática que ha caracterizado esta 


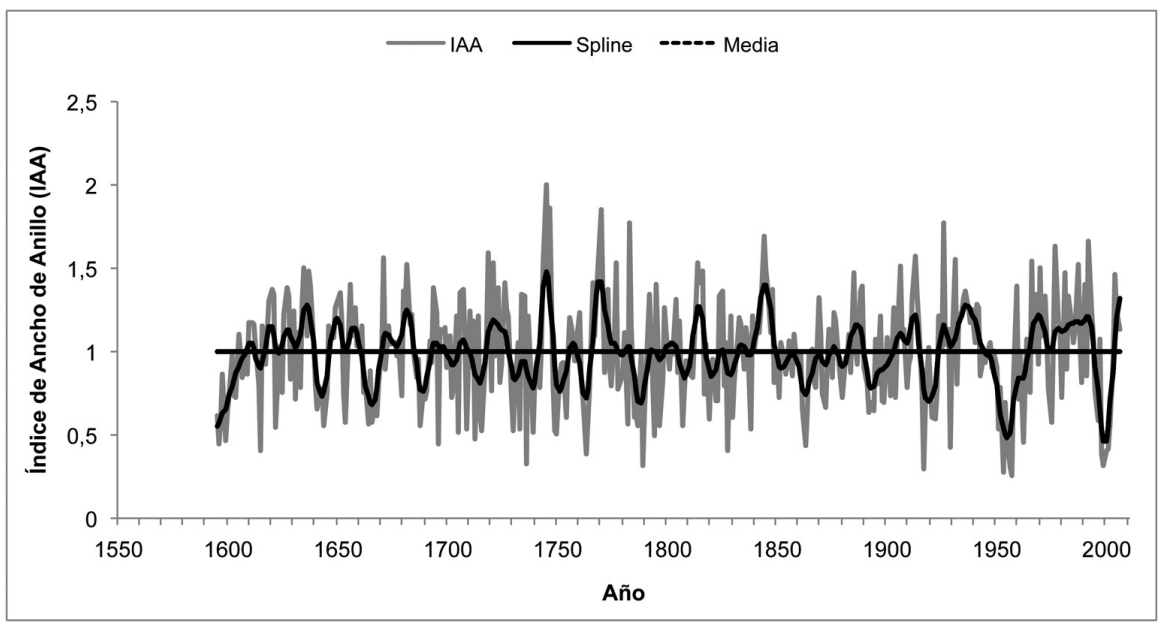

Figura 3. Cronología estándar de anillo total representativa de la parte baja de la Sierra de Zapalinamé mostrando alta variabilidad hidroclimática, lo que sugiere la presencia de sequías de gran magnitud; particularmente en las décadas de 1950 y 2000.

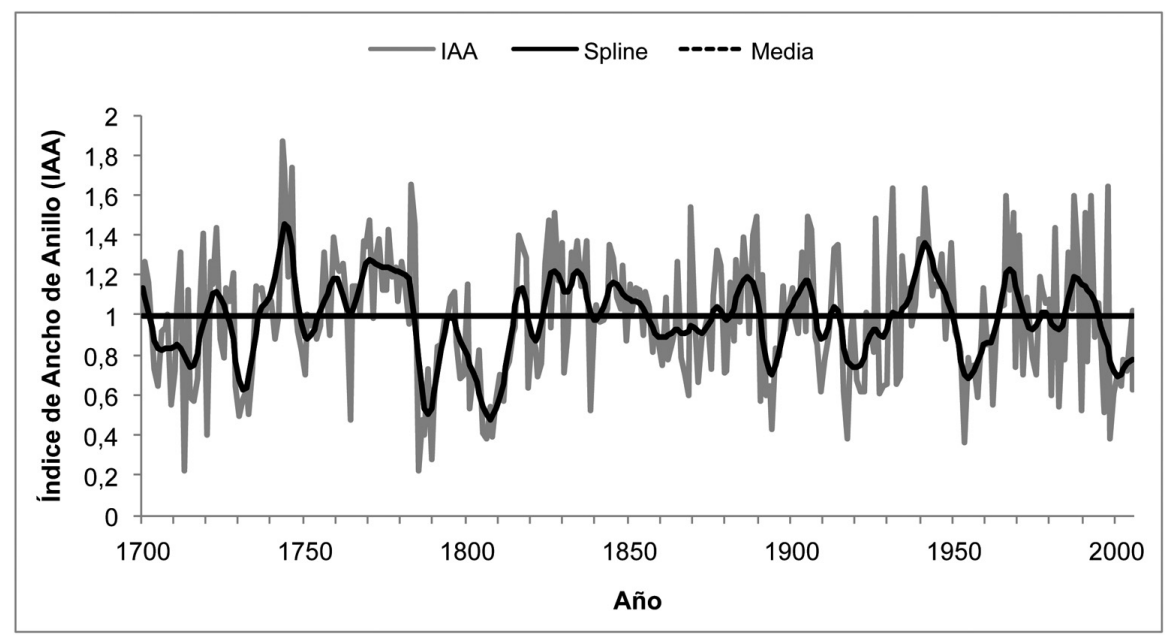

Figura 4. Cronología estándar de anillo total representativa de la parte intermedia de la Sierra de Zapalinamé mostrando alta variabilidad hidroclimática en esta parte de la cuenca y sequías de gran magnitud a mediados y finales del siglo XX (décadas de 1920,1950 y 2000); principios del XIX (1800) y mediados y finales del siglo XVIII

$(1730,1790)$. 


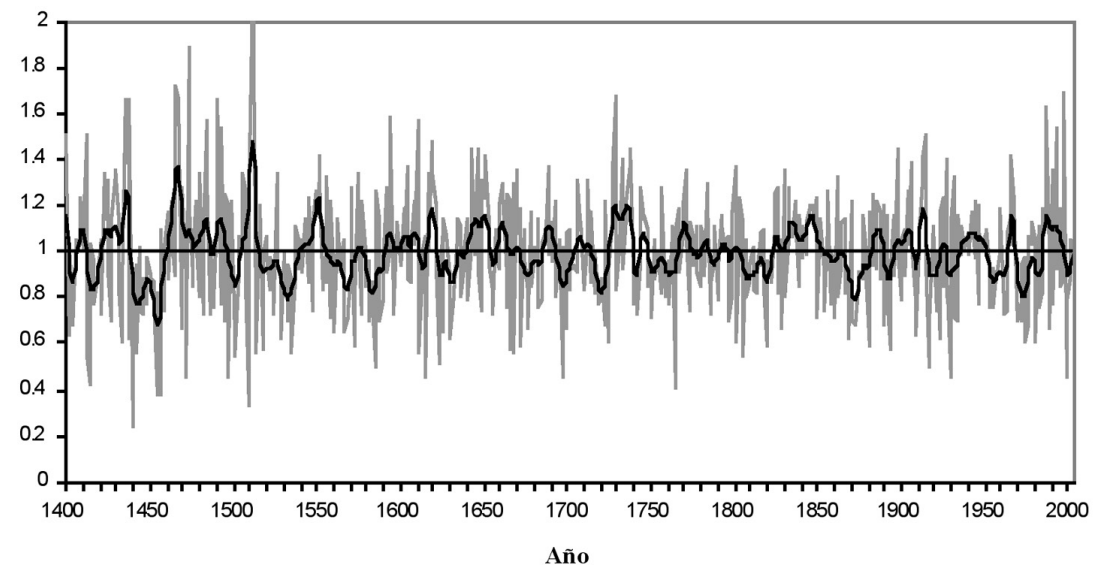

Figura 5. Cronología estándar de anillo total representativa de la parte alta de la Sierra de Zapalinamé mostrando alta variabilidad hidroclimática en esta sección de la montaña y sequías de gran magnitud, a lo largo de más de 600 años de la serie de tiempo desarrollada

región en los últimos cuatro siglos. Con base en los datos climáticos regionales (1950-1999) y la serie de tiempo generada, el programa PRECON determinó que el periodo de lluvia que influye de manera significativa $(p<0,05)$ en el crecimiento de la especie, es el periodo invierno-primavera (enero-julio). Al relacionar la cronología de madera temprana con datos instrumentales regionales de precipitación estacional enero-julio para el periodo 1950-1999, se encontró una respuesta significativa entre ambas variables con una correlación de $0,73(p<0,001)$, lo que sugiere potencial adecuado para la reconstrucción de variables climáticas, particularmente precipitación.

El modelo utilizado para la reconstrucción de lluvia incluyó el periodo total de datos disponibles (1950-1999) y los índices de ancho de anillo para el mismo periodo (tablas 6 y 7). Una vez aplicado el modelo, éste se calibró con los datos observados y los reconstruidos. Los resultados del modelo indican una correlación de $r=0,73\left(r^{2}=0,53, p<0,001\right)$ entre la precipitación observada y la precipitación reconstruida, lo que implica que el índice de ancho de anillo explica por sí solo $53 \%$ de la variabilidad de precipitación estacional (Figura 6). La subrutina VERIFY5 de la base de Programas Dendrocronológicos de la Universidad de Arizona (DPL, por sus siglas en inglés) indicó que la calibración pasó la prueba de significancia $(p<0,05)$ para la correlación, reducción de error, valor de " $\mathrm{t}$ " y primera diferencia significativa. El modelo de regresión obtenido para el periodo 1950-1999 se consideró estadísticamente válido para reconstruir el periodo total de la serie dendrocronológica.

El modelo bivariado utilizado fue el siguiente:

$$
Y t=17,3176+206,9509 * X t
$$

donde: $\mathrm{Yt}=$ Valor de precipitación reconstruida enero-julio para un año específico $(\mathrm{mm})$. $\mathrm{Xt}=$ Índice de ancho de anillo. 
Tabla 6. Modelo lineal generado para la reconstrucción estacional de precipitación invierno-primavera.

\begin{tabular}{lccccc}
\hline Periodo & $R^{2}$ & Coeficientes & $\begin{array}{c}\text { Error } \\
\text { Estándar }\end{array}$ & $\begin{array}{c}\text { Prueba de } \\
\text { " } t \text { “ }\end{array}$ & Probabilidad \\
\hline \multirow{2}{*}{$1950-1999$} & 0,56 & a 17,3176 & 27,72 & 0,625 & 0,5353 \\
& & b 206,9509 & 27,35 & 7,568 & 0,0000 \\
\hline
\end{tabular}

Tabla 7. Análisis de varianza del modelo de reconstrucción generado.

\begin{tabular}{lccccc}
\hline Periodo & Suma de cuadrados & $d f$ & Cuadrados medios & $f$ & $p$ \\
\hline Regresión & 231041,6 & & 231041,6 & 57,274 & 0,00000 \\
& & 1 & & & \\
Residual & 181528,1 & 45 & 4034,0 & & \\
Total & 412569,7 & & & & \\
\hline
\end{tabular}

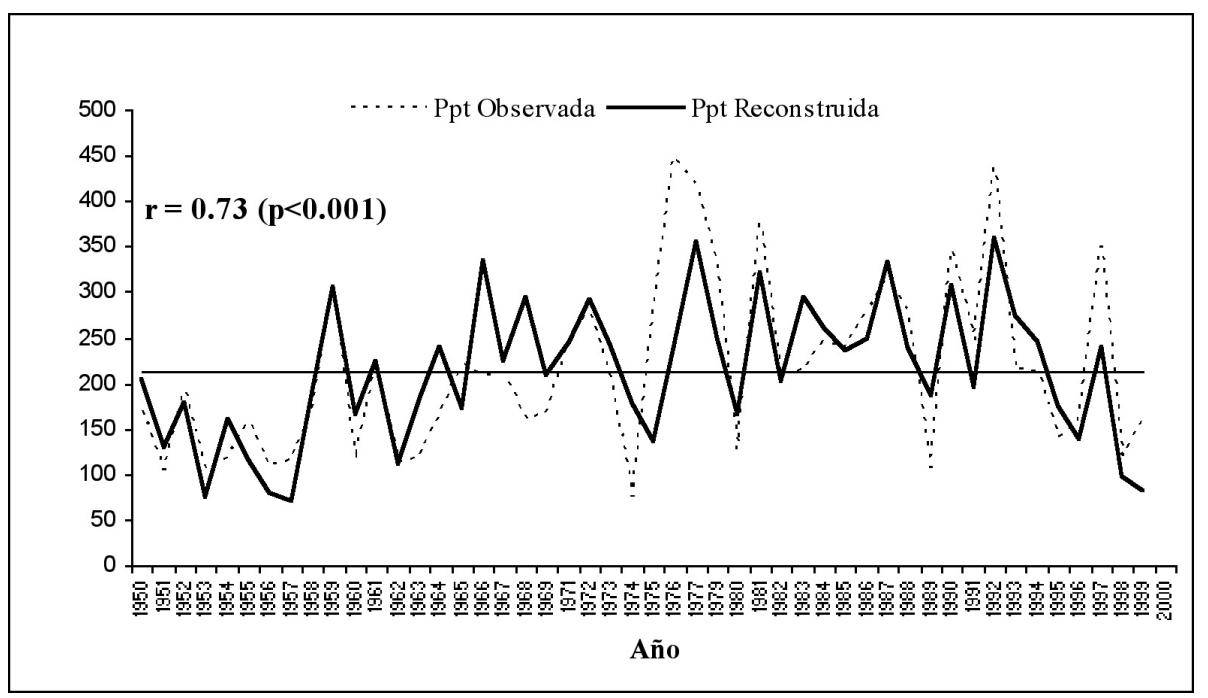

Figura 6. Calibración entre precipitación observada y reconstruida $r=0,73\left(R^{2}=0,53, p<0,001\right)$. 
La reconstrucción de más de 400 años de precipitación indica que SZA ha estado sujeta a una gran variabilidad hidroclimática histórica, pero a diferencia de otras reconstrucciones, los periodos de sequía más severa se presentaron a lo largo del siglo $\mathrm{XX}$, es decir, las décadas de 1920, 1950 y 1990-2000 (Figura 7). Los periodos secos detectados en esta reconstrucción fueron variables en términos de tiempo e intensidad $y$ muchos de ellos provocaron hambrunas generalizadas en la región e incluso a nivel nacional (Florescano, 1980). Periodos con limitada precipitación se detectaron en la reconstrucción para los periodos 1595-1618, 1641-1646, 16621670, 1687-1694, 1730-1743, 1784-1794, 1891-1896, 1917-1934, 1945-1962 у 1993-2004. Los periodos húmedos fueron menos frecuentes y se presentaron en las décadas de 1650, 1680, 1730, 1750, $1770,1840,1880,1930$ y 1980. La información histórica para la región se abocó en gran medida al análisis del impacto de eventos secos y existe poca información relacionada con periodos húmedos, no obstante eventos húmedos similares se detectaron en reconstrucciones dendroclimáticas para otras zonas del noreste de México, lo que implica que eventos climáticos de amplia extensión pudieran influenciar este comportamiento (Villanueva et al., 2007).

\section{Impacto del Niño Oscilación del Sur en SZA}

La asociación entre la variable índice dendrocronológico generado y el Índice de Lluvia Tropical, un estimativo del Niño Oscilación del Sur (Wright, 1979), ambos para el periodo 1895-1994, fue significativa $(p<0,05)$ particularmente para los primeros 20 años de comparación (1895-1914); posteriormente esta asociación decae y se torna no significativa $(p>0,05)$ para el resto de los subperiodos. Una respuesta similar se observa para la parte media y alta de la SZA (Tabla 8 y Figura 8).

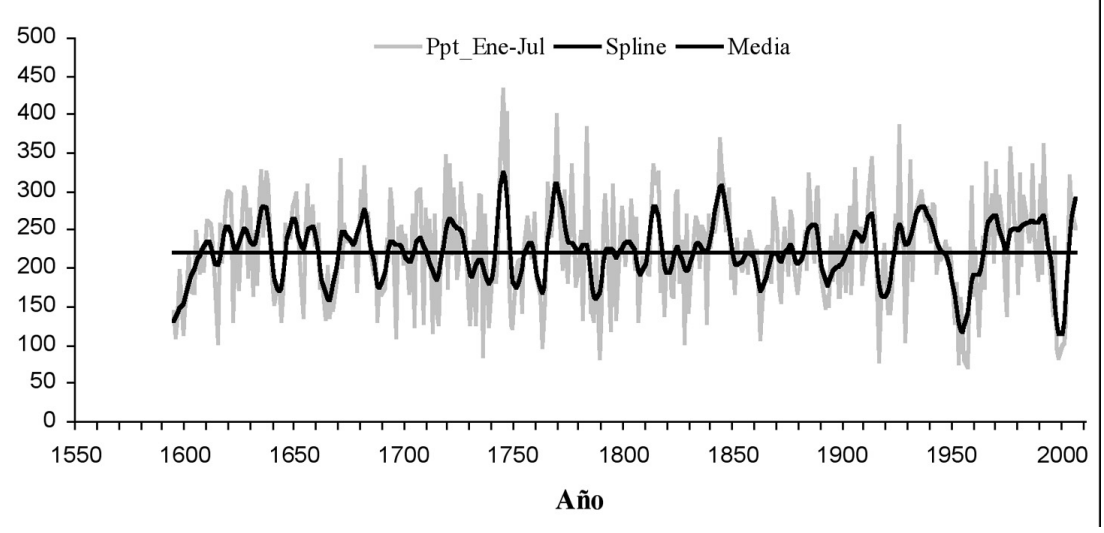

Figura 7. Reconstrucción regional de precipitación estacional (enero-julio) para la planicie de la Sierra de Zapalinamé, Coahuila. Los valores reconstruidos anuales se muestran en color gris. La línea en negritas es una curva flexible ("spline") que resalta eventos de baja frecuencia a nivel década. La línea horizontal obscura es la media de la precipitación reconstruida y valores que caen a una desviación estándar $(64,5 \mathrm{~mm})$ por abajo o encima de esta línea se consideran secos o húmedos, respectivamente. 
Tabla 8. Correlaciones obtenidas entre los índices dendrocronológicos generados a lo largo del gradiente en SZA y el Índice de Lluvia Tropical.

\begin{tabular}{|c|c|c|c|}
\hline \multirow[t]{2}{*}{ Periodo } & \multicolumn{3}{|c|}{ Cronologías representativas del gradiente altitudinal de SZA } \\
\hline & Alta1 & Media1 & Baja1 \\
\hline $1895-1914$ & $0,72(p<0.001)$ & $0,66(p<0.002)$ & $0,56(p<0.01)$ \\
\hline $1915-1934$ & $0,34(p>0.05)$ & $0,41(p>0.05)$ & $0,39(p>0.05)$ \\
\hline $1935-1954$ & $0,14(p>0.05)$ & $0,31(p>0.05)$ & $0,06(p>0.05)$ \\
\hline $1955-1974$ & $0,39(p>0.05)$ & $0,40(p>0.05)$ & $0,42(p>0.05)$ \\
\hline $1975-1994$ & $0,34(p>0.05)$ & $0,41(p>0.05)$ & $0,48(p<0.02)$ \\
\hline
\end{tabular}

$1 \quad$ Valores de correlación con su valor de probabilidad (entre paréntesis); las correlaciones significativas se presentan en negritas

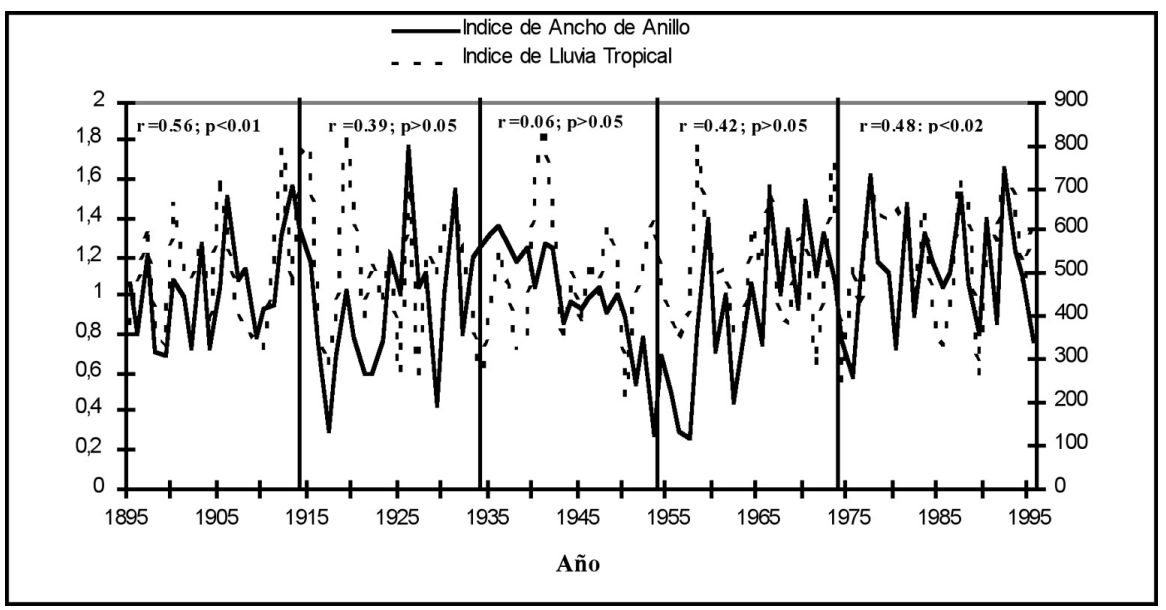

Figura 8. Relación entre la cronología representativa de la parte baja de SZA y el TRI.

La línea sólida obscura es el valor anual del índice de ancho de anillo y la línea quebrada representa el valor estacional enero-julio del índice de lluvia tropical (TRI). 


\section{DISCUSIÓN}

Asociación entre cronologías representativas del gradiente altitudinal

La asociación entre cronologías representativas de la parte alta, media y baja del gradiente altitudinal de la SZA fue significativa tanto para el periodo común de comparación (17002002) como para subperiodos de 50 años; aunque es de notar que la mayor correlación entre cronologías se obtuvo para el periodo de 1850 a 1999 , lo que probablemente sea un reflejo del mayor número de muestras involucrado en la estimación de los índices dendrocronológicos para dicho periodo (Tabla 4).

La respuesta detectada entre cronologías, implica que el clima en esta región parece tener un efecto común en el desarrollo de las especies arbóreas en esta montaña. Las variaciones detectadas entre cronologías pudieran atribuirse al efecto del gradiente orográfico que favorece para un mismo evento meteorológico una mayor precipitación en elevaciones superiores, aunque inferior en la parte media y baja de la montaña. La mayor precipitación en elevaciones superiores, trae consigo una mayor disponibilidad de agua para el crecimiento de las especies de bosque ahí presentes y por ende menor fluctuación en los crecimientos anuales o índices dendrocronológicos que indican una desviación estándar de 0,25 , en comparación a la parte baja dominada por pino piñonero, cuyos índices poseen una desviación estándar de 0,31 (Tabla 5), es decir, mayor variación en crecimiento y mayor sensibilidad a las variaciones en disponibilidad de agua que fluctúa a través de los años.

Al fijar curvas flexibles decenales a las cronologías regionales para resaltar eventos de baja frecuencia, estas indicaron concordancia en términos de responder simultáneamente a eventos secos y húmedos; aunque es de notar la mayor sensibilidad climática a eventos secos de las cronologías de la parte media y baja en relación con la de la parte alta (Figura 9). De esta manera, se observa

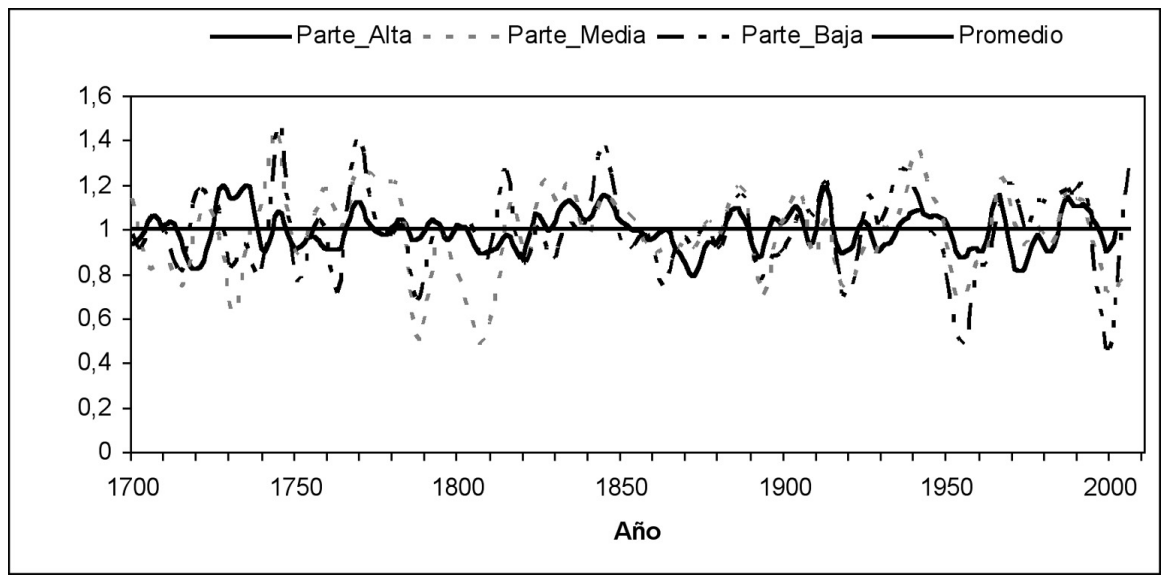

Figura 9. Curvas decenales flexibles ajustadas a los índices dendrocronológicos anuales para resaltar eventos de baja frecuencia a lo largo del gradiente altitudinal. Valores abajo del promedio se consideran secos y arriba húmedos. 
que eventos secos de baja frecuencia fueron detectados en las curvas flexibles para todas las cronologías en las décadas de 1990, 1950, 1920, 1890 y 1860.

En general, los eventos de baja frecuencia se presentaron simultáneamente entre cronologías, aunque las cronologías de la parte media y baja mostraron mayor sensibilidad a periodos de escasa precipitación, es decir décadas de 1990, 1950, 1920, 1890, 1860 y 1790; lo que indica que los árboles en estos sitios se ven sujetos a una menor disponibilidad de agua y por ende poseen una mayor sensibilidad climática o respuesta a periodos húmedos o secos.

\section{Reconstrucción de la precipitación}

El crecimiento de anillo total, respondió de manera significativa $(p<0,05)$ al periodo de precipitación invierno-primavera, coincidiendo de esta manera con lo señalado por Michaelsen (1989), Stahle y Cleaveland (1993), Stahle et al. (1999), y Cerano (2004), Constante (2007), Villanueva et al. (2007) y Santillán (2008), quienes indican que las cronologías de anillos de árboles procedentes del norte de México, responden de manera significativa a la precipitación estacional invierno-primavera.

La cronología explica $53 \%$ de la variabilidad en precipitación que caracteriza a la parte baja de la SZA, porcentaje que se considera satisfactorio en términos de respuesta climática de anillos de árboles y del periodo reconstruido. Más del $50 \%$ de la precipitación en esta montaña ocurre en el verano, sin embargo, en este estudio no se detectó una respuesta significativa para esta estación, posiblemente debido a que mucha de esta precipitación es de alta intensidad, satura la capacidad de almacenamiento del suelo y mucha de ella se pierde por escorrentía; además de ocurrir en una época en que el crecimiento radial de la especie casi ha concluido (Villanueva et al., 2007).

Gran parte de los eventos de baja frecuencia reconstruidos en este estudio se encuentran también presentes en reconstrucciones previas realizadas para el norte y centro de México (Cleaveland et al., 2003; Cerano, 2004; Villanueva et al., 2007); así como en archivos históricos de carácter regional y nacional; casos específicos son las sequías de las décadas de $1670,1720,1760,1790,1820,1860$, 1890, 1920, 1950, 1970 y 2000 (Figura 10). Las correlaciones entre reconstrucciones fueron altamente significativas $(p<0,0001)$ aún para diferentes regiones, aunque las más altas correlaciones se obtuvieron al comparar reconstrucciones climáticas para una región o sistema montañoso en particular, caso específico reconstrucciones para la Sierra Madre Oriental en comparación con aquellas de la Sierra Madre Occidental.

La sequía de las décadas de 1450 a 1460 impactó severamente al Valle de México y produjo hambruna generalizada en todo el país (García, 1993). Las sequias de 1750 y 1760 provocaron escasez de maíz en la región de Saltillo; así como los años de 1784 a 1786, que originó muerte entre la población debido a desnutrición (Florescano, 1980; García, 1997). La crisis agrícola nacional de 1801 a 1802 y 1807 a 1810 provocó escasez de granos básicos en Saltillo, algo similar se presentó en 1820,1831, 1850 y década de 1860, año de 1877 (relacionado con el Niño), 1884-1885, 1892, 1896, 1923, 1925, 1927, 1951, 1960-1964 y 1993. La sequía más reciente del periodo 19932004, no obstante de ser una de las más severas en los últimos 400 años para esta región, aparentemente no ha tenido el impacto de algunas sequías previas, lo cual puede ser debido a que esta sequía 
se ha mitigado mediante la sobreexplotación de los mantos acuíferos para subsanar la falta de agua, importación de granos básicos de otras regiones productoras del país o bien del extranjero, subsidios gubernamentales a productos básicos, diversificación de actividades productivas y migración de la población a sitios con menor afectación climática, entre otras acciones (Stahle et al., 2009). Los modelos de circulación general indican una tendencia a sequías más recurrentes en el norte de México (Seager et al., 2007, 2009), lo que implica mayores problemas para subsanar el déficit de recursos hídricos ante una población creciente que demanda mayores volúmenes de agua para diversos usos; por lo anterior es importante desarrollar planes a largo plazo de manejo sustentable de los recursos hídricos.

\section{Impacto de ENSO en SZA}

La influencia de El Niño Oscilación del Sur (ENSO), medida a través del índice de lluvia tropical (TRI) indicó poca influencia en las condiciones del clima prevalecientes a lo largo de la SZA. La mayor influencia de este fenómeno en las condiciones climáticas del área de estudio ocurrió para el periodo 1895 a 1914, cuando se obtuvieron correlaciones de $0,72,0,66$ y 0,56 para la parte alta, media y baja de la montaña, respectivamente. Posteriormente, las correlaciones no fueron significativas (Tabla 8, Figura 8), posiblemente debido a la variabilidad natural de este fenómeno y a cambios en su comportamiento producto de acciones antropogénicas y de su impacto en el calentamiento global.

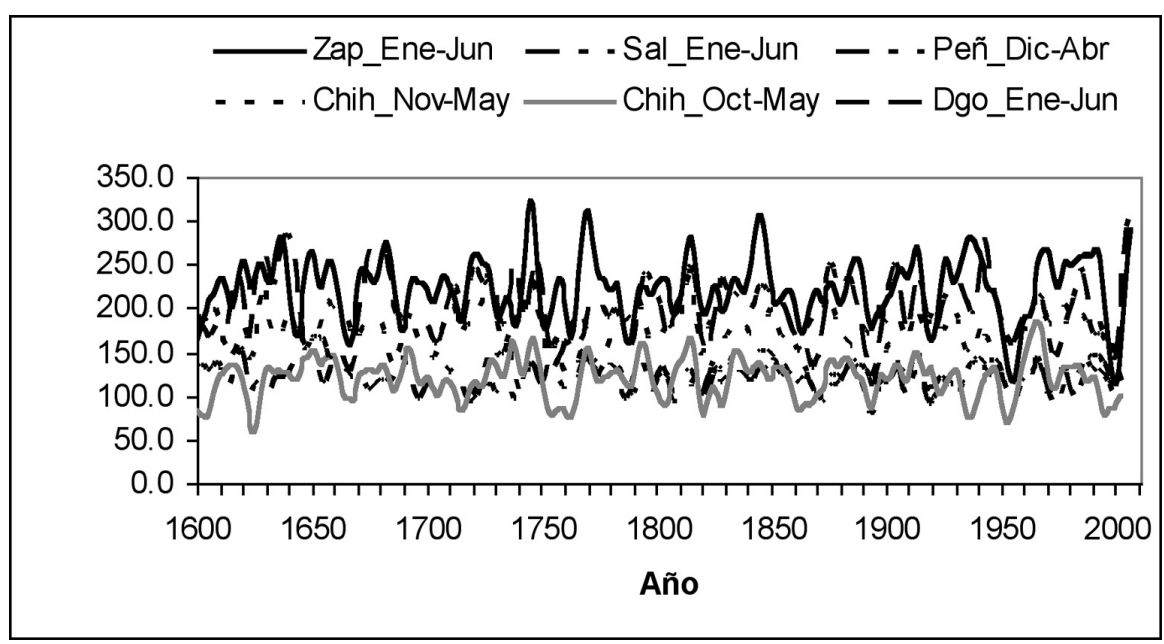

Figura 10. Eventos de baja frecuencia a nivel década para reconstrucciones estacionales de precipitación para el norte y noreste de México. Las reconstrucciones indican eventos comunes, generalmente de alta intensidad, que pueden estar ligados a patrones atmosféricos circulatorios que impactan grandes regiones de México, caso específico El Niño Oscilación del Sur. Variaciones entre reconstrucciones se atribuyen a condiciones climáticas regionales y a la influencia dominante de otros fenómenos atmosféricos; caso concreto, frentes fríos, huracanes, tormentas tropicales, particularmente para el noreste de México. 
Otros fenómenos atmosféricos como nortes, depresiones tropicales, huracanes, etc., que afectan la Sierra Madre Oriental, probablemente ejerzan una influencia más significativa en el clima que caracteriza a esta región (Villanueva et al., 2007).

\section{CONCLUSIONES}

El entendimiento de la variabilidad hidroclimática es fundamental, para el manejo del agua en SZA, que en las últimas dos décadas se ha visto sujeta a una extrema presión social por los recursos hídricos disponibles para satisfacer los requerimientos agropecuarios, industriales y para consumo humano del núcleo poblacional de Saltillo, Coahuila y área conurbada. Las cronologías de anillos de árboles en SZA y montañas aledañas fueron sensitivas a la variabilidad hidroclimática que ha caracterizado esta región en los últimos 600 años.

La presencia de eventos secos y húmedos fue detectada por las cronologías en todo el gradiente altitudinal, aunque es de notar que la cronología de pino piñonero de la parte baja del gradiente fue la más sensible a periodos secos, lo cual tiene sentido, ya que la especie se desarrolló en sitios semiáridos con alta insolación y demanda evaporativa. Sequías de gran magnitud e intensidad se presentaron en todo el gradiente en las décadas de 1440 y 1450; 1530, $1590,1640,1660,1690,1710,1740$, $1750,1760,1790,1860,1890,1920$, 1950 y 2000; es decir, en periodos aproximados de 100 años con sequías intermedias cada 50 años. Algunos de los periodos secos fueron de mayor intensidad a los registrados en el siglo $X X$; sin embargo, la tendencia actual de calentamiento del planeta y el cambio climático global pudiera favorecer la presencia de eventos climáticos extremos y de mayor frecuencia y afectar la variabilidad hidroclimática histórica en ésta y otras regiones de México.

El impacto de ENSO no fue significativo en esta región y se presume que otros fenómenos atmosféricos como frentes fríos, huracanes y tormentas tropicales pudieran explicar con mayor detalle la climatología de esta montaña; no obstante es importante a corto plazo incrementar la red de cronologías de anillos de árboles en esta región, lo que permitiría tener un conocimiento más detallado de la variabilidad hidroclimática y el impacto más preciso de patrones circulatorios.

\section{AGRADECIMIENTOS}

Esta investigación fue posible gracias al financiamiento otorgado por el Instituto Interamericano para la Investigación del Cambio Climático (IAI), a través del proyecto CRN núm. 2047 Documentación, Entendimiento y Proyección de los Cambios en el Ciclo Hidrológico en la Cordillera Americana, a su vez financiado por el US/Nacional Science Foundation (Grant GEO-0452325).

\section{REFERENCIAS}

Cerano, P. J. 2004. Reconstrucción de 350 años de precipitación inviernoprimavera para Saltillo, Coahuila. Tesis Profesional. Departamento Forestal. Universidad Autónoma Agraria Antonio Narro. Saltillo, Coahuila, México, 152 p.

Cerano, P. J. 2008. Variabilidad climática, regímenes de incendios e influencia de patrones circulatorios para el suroeste de Chihuahua. Tesis de Maestría. Universidad Autónoma Chapingo, Unidad Universitaria de Zonas Áridas. Bermejillo, Durango, México, $118 \mathrm{p}$. 
Cleaveland, M. K., D.W. Stahle, M. D. Therrell, J. Villanueva-Díaz y B. T. Burns. 2003. Tree-ring reconstructed winter precipitation and tropical teleconnections in Durango, Mexico. Climatic Change 59: 369-388.

Comisión Nacional del Agua. 2002. Determinación de la disponibilidad del agua en el acuífero Saltillo-Ramos Arizpe, estado de Coahuila. CONAGUA-Gerencia de Aguas Subterráneas. México, D.F., 29 p.

Comisión Nacional del Agua. 2006. Statistics on water in Mexico. CONAGUA. Fourth Edition. México, D. F., 186 p.

Constante García, V. 2007. Reconstrucción de la precipitación inviernoprimavera para los últimos cuatro siglos en el ejido Cuauhtémoc, Saltillo, Coahuila. Tesis profesional. Ingeniero Forestal, Departamento Forestal, UAAAN, Buenavista, Saltillo, Coahuila, $72 \mathrm{p}$.

Cook, E. R. 1987. The decomposition of tree-ring series for environmental studies. Tree-Ring Bulletin 47: 37-59.

Cook, E. R. y Peters, K. 1981. The smoothing spline: a new approach to standardizing forest interior tree-ring width series for dendroclimatic studies: Tree-Ring Bulletin 41: 45-53.

ERIC II. 2000. Instituto Mexicano de Tecnología del Agua. Extractor Rápido de Información Climatológica.

Florescano, E. 1980. Análisis histórico de las sequías en México. Secretaría de Agricultura y Recursos Hidráulicos, Comisión del Plan Nacional Hidráulico, México, D. F., 158 p.

Fritts, H. C. 1991. Reconstructing largescale climatic patterns from tree-ring data. University of Arizona Press. Tucson, $286 \mathrm{p}$.

Fritts, H. C. 1999. An Empirical Model of the Tree-Ring Response to Monthly variations in climate. Laboratory of Tree-Ring Research University of Arizona, Tucson, Arizona, USA.

García, A. V. 1993. Las sequías históricas de México. Desastres y Sociedad 1(1): 1-18.

García, E. 2004. Modificaciones al sistema de clasificación climática de Köppen. Instituto de Geografía de la UNAM. $5^{\circ}$. ed., México, D. F., 90 p.

García H. A. 1997. Alternativas ante las sequías de 1789-1810 en la villa de Saltillo, Coahuila, México. En: García Acosta V. (coord.). Historia y desastres en América Latina. Vol. II. Red de Estudios Sociales en América Latina, 148-169.

Grissino-Mayer, H. D. 2001. Evaluating crossdating, accuracy: a manual and tutorial for the computer program COFECHA. Tree-Ring Research 57(2): 205-221.

Holmes, R. L. 1983. Computer-assisted quality control in tree-ring dating and measurement. Tree-Ring Bulletin 43: 69-78.

Marroquín, S. J. y L. Arce. 1985. Las unidades fisonómicas-florísticas del Cañón de San Lorenzo, Saltillo, Coah., México, Biota 10(4): 369-393.

Michaelsen, J. 1989. Long period fluctuations in El Niño amplitude and frequency reconstructed from tree rings. In: Peterson, D. H. (ed.). 1989. Aspects of Climate Variability in the Pacific and the Western Americas. AGU, Washington, D. C. Geophysical Monograph 55, pp. 69-74. 
Robinson, W. J. y R. Evans. 1980. A microcomputer-based tree-ring measuring system. Tree-Ring Bulletin 40: 59-64.

Santillán, H. M. 2008. Dendrocronología de diez poblaciones de Pinus pinceana Gordon de la Sierra Madre Oriental. Tesis de Maestría. Universidad Autónoma Agraria "Antonio Narro". Departamento Forestal. Buenavista, Saltillo, Coahuila, 120 p.

Seager R., M. Ting, I. Held, Y. Kushnir, J. Lu, G. Vecchi, H. Huang, N. Harnik, A. Leetmaa, N. Lau, C. Li, J. Velez y N. Naik. 2007. Model projections of a imminent transition to a more arid climate in southwestern North America. Science 316: 1181-1184.

Seager, R., M. Ting., M. Davis, M. Cane, N. Nike, J. Nakumara, C. Lie, E. Cook, y D. W. Stahle. 2009. Mexican drought: an observational modeling and tree ring study of variability and climate change. Atmosfera 22(1): 1-31.

Stahle, D. W. y M. K. Cleaveland. 1993. Southern oscillation extremes reconstructed from tree-rings of the Sierra Madre Occidental and Southern Great Plains. Journal of Climate 6: 129-140.

Stahle, D. W., M. K. Cleaveland, M. D. Therrell, y J. Villanueva-Díaz. 1999. Tree-ring reconstruction of winter and summer precipitation in Durango, Mexico, for the past 600 years. 10th Conference of Global Change Studies. Preprint volume, American Meteorological Society, 79th Annual Meeting. January 1015. Dallas, Texas.

Stahle, D. W., E. R. Cook, J. VillanuevaDiaz, F. K. Fye, D. J. Burnett, R. D. Griffin, R. Acuña-Soto, R. Seager y R.R. Heim Jr. 2009. Early 21stcentury drought in Mexico. Eos 90(17): 89-90.

Stat soft Inc. 2000. STATISTICA Kernel release $5.5 \mathrm{~A} .1984-2000$.

Stokes, M. A. y T .L. Smiley. 1968. An introduction to tree-ring dating. The University of Chicago, $73 \mathrm{p}$.

Universidad Autónoma Agraria "Antonio Narro" (UAAAN). 1998. Programa de manejo de la zona sujeta a conservación ecológica "Sierra de Zapalinamé". Saltillo, Coahuila, 214 p.

Villanueva Díaz, J., J. Cerano P., D. W. Stahle, M. D. Therrell, L. Vázquez S., R. Morán M., y B. H. Luckman. 2006. Árboles viejos del centro-norte de México: importancia ecológica y paleoclimática. Folleto científico 20. INIFAP-CENID-RASPA, Gómez Palacio, Durango, $46 \mathrm{p}$.

Villanueva-Díaz, J.; D. W. Stahle, B. H. Luckman, J. Cerano-Paredes, M. D. Therrell y M. K. Cleaveland. 2007. Winter-spring precipitation reconstructions from tree rings for northeast Mexico. Climatic Change 83: 117-131.

Wright, P. B. 1979. Persistance of rainfall anomalies in the Central Pacific. Nature 277: 371-374.

Manuscrito recibido el 29 de julio de 2008

Aceptado el 27 de mayo de 2009

Este artículo se debe citar como: Villanueva-Díaz, J.; J. Cerano-Paredes; V. Constante-García; P. Z. Fulé y E. Cornejo-Oviedo. 2009. Variabilidad hidroclimática histórica de la Sierra de Zapalinamé y disponibilidad de recursos hídricos para Saltillo, Coahuila. Madera y Bosques 15(3): 45-64. 